Proyecciones

Vol. 23, No 2, pp. 111-121, August 2004.

Universidad Católica del Norte

Antofagasta - Chile

\title{
ON AUTOMATIC SURJECTIVITY OF SOME ADDITIVE TRANSFORMATIONS
}

\author{
MUSTPHA ECH-CHÉRIF EL KETTANI \\ EL HOUCINE El BOUCHIBTI \\ Universidad de Fes, Maroc
}

Received: May 2003. Accepted: May 2004.

\begin{abstract}
Let $X$ be an infinite dimensional Banach space and let $\Phi: B(X) \longrightarrow$ $B(X)$ be a spectrum preserving additive transformation. We show that if the image of quasi-nilpotent operators contains all quasi-nilpotent operators, then $\Phi$ is an automophism or an antiautomorphism of $B(X)$.
\end{abstract}




\section{Introduction}

Throughout this paper we will denote by $X$ an infinite dimensional complex Banach space, $X^{\prime}$ the dual of $X, B(X)$ the algebra of all linear bounded operators on $X$, and $Q N(X)$ the set of quasi-nilpotent operators in $B(X)$. It seems that in the last few years there has been a growing interest in problems of caracterizing transformations preserving some sets between the algebras of linear bounded operators over infinite dimensional Banach spaces, we can see for exemple [1], [5], [7], [8], [10], [11], [13]. It has been proved by B. Aupetit [3] that if $\Phi: B(X) \longrightarrow B(X)$ is a spectrum preserving surjective additive transformation, then $\Phi$ is an automorphism or an antiautomorphism of $B(X)$. He asked the question what happens if such transformation is not surjective. In this paper we propose to have the same result of B. Aupetit by the same hypotheses but we assume only that the image of quasi-nilpotent operators contains all quasi-nilpotent operators.

Let us fix some notations. For every $T \in B(X)$ we denote by $\sigma(T)$ and $\rho(T)$ the spectrum and the spectral radius of $T$ respectively. Every operator on $X$ of rank one can be written as $x \otimes f$ for some $x \in X-\{0\}$ and $f \in X^{\prime}-\{0\}$. We recall that $x \otimes f$ is defined by $(x \otimes f) y=f(y) x$ for $y \in X$. The operator $x \otimes f$ is a quasi-nilpotent operator if and only if $f(x)=0$. For $x, y \in X$ and $f, g \in X^{\prime}$, the operator $x \otimes f+y \otimes g$ is of rank one if and only if either $x$ and $y$ are linearly dependent or $f$ and $g$ are linearly dependent ( see [10] and [11] ). We denote by $\lambda$ the operator $\lambda . I$ for every $\lambda \in \mathbf{C}$ and by $F_{1}(X)$ the set of rank one operators of $B(X)$.

\section{Main results}

The aim of this work is to prove the following results:

Theorem 2.1. Let $\Phi: B(X) \longrightarrow B(X)$ be a spectrum preserving additive transformation such that $Q N(X) \subset \Phi(Q N(X))$. Then either

i) $\Phi(T)=A T A^{-1}$, for every $T \in B(X)$, where $A: X \longrightarrow X$ is a bounded bijective linear operator; or

ii) $\Phi(T)=B T^{*} B^{-1}$ for every $T \in B(X)$, where $B: X^{\prime} \longrightarrow X$ is a bounded bijective linear operator. In this case $X$ must be reflexive.

Corollary 2.1. Let $\Phi: B(X) \longrightarrow B(X)$ be a spectrum preserving additive transformation such that $Q N(X) \subset \Phi(Q N(X))$. Then $\Phi$ is surjective. 
In order to prove those results we need some lemmas.

The following lemma is due to B. Aupetit ( [3], Corollaire 2.4 ).

Lemma 2.1. Let $\Phi: B(X) \longrightarrow B(X)$ be a spectrum preserving additive transformation. Then $\operatorname{rank}(T) \leq \operatorname{rank} \Phi(T)$ for every $T \in B(X)$. In particular $\Phi$ is injective.

The following lemma is a result of A. Jafarian et A. R. Sourour ( [7], Lemma 4 ).

Lemma 2.2. Let $x \in X, f \in X^{\prime}, T \in B(X)$ and $\lambda \notin \sigma(T) . \lambda \in \sigma(T+x \otimes$ $f)$ if and only if $f\left((\lambda-T)^{-1} x\right)=1$.

Lemma 2.3. Let $R \in F(X)$. The following conditions are equivalent:

1) $\operatorname{rank}(R)=1$,

2) For every $Q \in Q N(X)$. We have, $\rho(Q+R) \neq 0$ implies that there exists $r \in \mathbf{Q}-\{0,1\}$ such that $\rho(Q+r R) \neq 0$.

Proof We show that 1) implies 2). Let us consider $Q \in Q N(X)$ and the operator $R$ of the form $R=x \otimes f$ where $x \in X-\{0\}$ and $f \in X^{\prime}-\{0\}$.

For every $\lambda \in \mathbf{C}^{*}$.

The resolvent $\lambda \longmapsto(\lambda-Q)^{-1}$ is holomorphic from $\mathbf{C}^{*}$ to $B(X)$ and $(\lambda-Q)^{-1}=\sum_{k=0}^{+\infty} \lambda^{-k-1} Q^{k}$, so the function $\lambda \longmapsto(\lambda-Q)^{-1} x$ is holomorphic from $\mathbf{C}^{*}$ to $X$ and $(\lambda-Q)^{-1} x=\sum_{k=0}^{+\infty} \lambda^{-k-1} Q^{k} x$. Therefore the function $\lambda \longmapsto f\left((\lambda-Q)^{-1} x\right)$ is holomorphic from $\mathbf{C}^{*}$ to $\mathbf{C}$ and $(\lambda-Q)^{-1} x=\sum_{k=0}^{+\infty} \lambda^{-k-1} Q^{k} x$, [ see [4], p:38-39 ]. 0 is not a removable singularity of the function $\lambda \longmapsto f\left((\lambda-Q)^{-1} x\right)$ because the Laurent's serie development $f\left((\lambda-Q)^{-1} x\right)=\sum_{k=0}^{+\infty} \lambda^{-k-1} f\left(Q^{k} x\right)$ has not a regular part ( we can also see that 0 is not a removable singurarity from the fact that the resolvent is not an entire function over $\mathbf{C})$. So 0 is either a pole or an essentiel singularity of the function $\lambda \longmapsto f\left((\lambda-Q)^{-1} x\right)$.

In the case where 0 is a pole of the function $f\left((\lambda-Q)^{-1} x\right)$, we establish that its principal part of the Laurent's serie development has a finite number of terms, so there exists an integer $n \in \mathbf{N}$ such that $f\left(Q^{m} x\right)=0$ for every $m \geq n$. If $\rho(Q+R) \neq 0$ there exists a non zero complex number $\mu \in \sigma(Q+R)$. By Lemma 2.2 we have $f\left((\mu-Q)^{-1} x\right)=1$ $(*)$. Let us define the polynomial $p(z)$ by $p(z)=\sum_{k=1}^{n-1} f\left(Q^{k} x\right) z^{k+1}$. For 
every $z \in \mathbf{C}^{*}$ we have $(z-Q)^{-1}=\sum_{k=0}^{n-1} Q^{k} z^{-k-1}$, which implies that $\left(z^{-1}-Q\right)^{-1}=\sum_{k=0}^{n-1} Q^{k} z^{k+1}$ and therefore $\left(z^{-1}-Q\right)^{-1} x=\sum_{k=0}^{n-1} Q^{k} x z^{k+1}$, thus $p(z)=f\left(\left(z^{-1}-Q\right)^{-1} x\right)$ for every $z \in \mathbf{C}^{*}$. By $\left(^{*}\right)$ we have $p \neq 0$ which implies that there exists a non zero complex number $\lambda$ such that $p(\lambda)=\frac{1}{2}$, it holds that $2 f\left(\left(\lambda^{-1}-Q\right)^{-1} x\right)=1$ and therefore $\lambda^{-1} \in \sigma(Q+2 R)$. Thus $\rho(Q+2 R) \neq 0$.

In the case where 0 is an essentiel singularity of the function $\lambda \longmapsto$ $f\left((\lambda-Q)^{-1} x\right)$. Picard's " Big " Theorem asserts that, in a neighbourhood of an essentiel singularity, an analytic function assumes all values, with at most one possible exception, infinitely often. Thus, there exists $\alpha \in \mathbf{C}^{*}$ and $r \in \mathbf{Q}^{*}$ such that $f\left((\alpha-Q)^{-1} x\right)=\frac{1}{r}$, which implies that $r f\left((\lambda-Q)^{-1} x\right)=1$ and therefore $\alpha \in \sigma(Q+r R)$. So, $\rho(Q+r R) \neq 0$.

To prove the reverse implication we follow a similar approch of the $\breve{S}$ emrl's one [11]. We consider a quasi-nilpotent operator $Q$ of rank greater than one. Such an operator has a matrix representation

$$
\left(\begin{array}{cc}
Q_{1} & Q_{2} \\
0 & Q_{3}
\end{array}\right) \text {, where } Q_{3} \text { is a quasi-nilpotent operator and } Q_{1} \text { is an }
$$
operator acting on a finite-dimensional space with a matrix representation $\left(\begin{array}{ccccccc}0 & 1 & 0 & . & . & . & 0 \\ 0 & 0 & 1 & . & . & . & 0 \\ . & . & . & . & . & . & . \\ . & . & . & . & . & . & . \\ . & . & . & . & . & . & . \\ 0 & 0 & 0 & . & . & . & . \\ 0 & 0 & 0 & . & . & . & 0\end{array}\right)$ or $\left(\begin{array}{cccc}0 & 1 & 0 & 0 \\ 0 & 0 & 0 & 0 \\ 0 & 0 & 0 & 1 \\ 0 & 0 & 0 & 0\end{array}\right)$ respectively. Let us consider an operator $R$ having a matrix representation $R=\left(\begin{array}{cc}R_{1} & 0 \\ 0 & 0\end{array}\right)$ with $R_{1}$ equal to $\left(\begin{array}{ccccccc}0 & -1 / r & 0 & . & . & . & 0 \\ 0 & 0 & 0 & . & . & . & 0 \\ . & . & . & . & . & . & . \\ . & . & . & . & . & . & . \\ . & . & . & . & . & . & . \\ 0 & 0 & 0 & 0 . & . & . & 0 \\ 1 / r & 0 & 0 & . & . & . & 0\end{array}\right)$ or $\left(\begin{array}{cccc}0 & -1 / r & 0 & 0 \\ 0 & 0 & 1 / r & 0 \\ 0 & 0 & 0 & 0 \\ 1 / r & 0 & 0 & 0\end{array}\right)$ respectively, with $r \in \mathbf{Q}-\{0,1\}$. We have $\rho(Q+R) \neq 0$ but $\rho(Q+r R)=0$. 
Lemma 2.4. Let $\Phi: B(X) \longrightarrow B(X)$ be a spectrum preserving additive transformation such that $Q N(X) \subset \Phi(Q N(X))$ and $R \in B(X)$. Then, $R$ is of rank one if and only if $\Phi(R)$ is of rank one.

Proof Let $R$ an operator of rank one in $B(X)$. By the Lemma 2.3, for every $Q \in Q N(X)$ satisfying $\rho(Q+2 R)=0$ we have $\rho(Q+R)=0$. The transformation $\Phi$ preserves the spectrum then for every $Q \in Q N(X)$ satisfying $\rho(\Phi(Q)+2 \Phi(R))=0$ we have $\rho(\Phi(Q)+\Phi(R))=0$. By the assumption $Q N(X) \subset \Phi(Q N(X))$, for every operator $Q \in Q N(X)$ satisfying $\rho(Q+2 \Phi(R))=0$ we have $\rho(Q+\Phi(R))=0$. Applaying the Lemma 2.3 again we conclude that $\Phi(R)$ is of rank one. To prove the reverse implication it suffices to applay Lemma 2.1.

Lemma 2.5. Let $\Phi: B(X) \longrightarrow B(X)$ be a spectrum preserving additive transformation such that $Q N(X) \subset \Phi(Q N(X))$. Then $\Phi(\lambda)=\lambda$ for every $\lambda \in \mathbf{C}$.

Proof If $\lambda=0$ we have $\Phi(0)=0$.

If $\lambda \neq 0$ we prove that for every $x \in X$ the vectors $\lambda x$ and $\Phi(\lambda) x$ are linearly dependent. Assume in the contrary that there exists $x \in X$ such that $\lambda x$ and $\Phi(\lambda) x$ are linearly independent. Let $U$ the linear span of $\{\lambda x$, $\Phi(\lambda) x\}$, and $W$ a closed complement of $U$. Define an operator $M$ on $X$ by

$$
\begin{aligned}
M \lambda x & =2 \lambda^{2} x-\lambda \Phi(\lambda) x \\
M \Phi(\lambda) x & =4 \lambda^{2} x-2 \lambda \Phi(\lambda) x \\
M w & =0 \text { pour } w \in W .
\end{aligned}
$$

Thus, we have that $M \in B(X)$ and $M^{2}=0$. By the assumption $Q N(X) \subset \Phi(Q N(X))$ there exists $Q \in Q N(X)$ such that $M=\Phi(Q)$. We have $\sigma(\lambda+Q)=\{\lambda\}$ and therefore $\sigma(\Phi(\lambda)+M)=\{\lambda\}$ this contradicts the fact that $(\Phi(\lambda)+M)(\lambda x)=2 \lambda(\lambda x)$. Thus, for every $x \in X$ the vectors $\Phi(\lambda) x$ and $\lambda x$ are linearly dependent. As $\Phi$ preserves the spectrum we have $\Phi(\lambda)=\lambda$.

Lemma 2.6. Let $\Phi: B(X) \longrightarrow B(X)$ be a spectrum preseving additive transformation such that $Q N(X) \subset \Phi(Q N(X))$. Then the restriction of $\Phi$ to $F_{1}(X)$ is 1-homogeneous on $F_{1}(X)$. ( i.e: $\Phi(\alpha R)=\alpha \Phi(R)$ for every $\alpha \in \mathbf{C}$ and $\left.R \in F_{1}(X)\right)$. 
Proof Since every operator of rank one can be written in the form $x \otimes f$ for some $x \in X$ and $f \in X^{\prime}$, it is enough to prove that $\Phi(\alpha x \otimes f)=\alpha \Phi(x \otimes f)$ for every $\alpha \in \mathbf{C}$.

Let us fix $\alpha \in \mathbf{C}^{*}, x \in X-\{0\}$ and $f \in X^{\prime}-\{0\}$. We start by the case where $f(x) \neq 0$, in which case we can take $f(x)=1$. By the Lemma 2.4 there exists $u \in X$ and $\varphi \in X^{\prime}$ such that $\Phi(x \otimes f)=u \otimes \varphi$. Because $\Phi$ preserves the spectrum we have $\varphi(u)=1$. Let us consider $v \in X-\{0\}$ et $\psi \in X^{\prime}-\{0\}$ such that $\varphi(v)=0$ and $\psi(u)=0$. Since the operators $v \otimes \varphi$ and $u \otimes \psi$ are quasi-nilpotent there exists $w, z \in X$ and $h, k \in X^{\prime}$ such that

$$
\Phi(w \otimes h)=v \otimes \varphi \text { and } \Phi(z \otimes k)=u \otimes \psi .
$$

By the Lemma 2.4 there exists $u_{\alpha} \in X$ and $\varphi_{\alpha} \in X^{\prime}$ such that $\Phi(\alpha x \otimes f)=$ $u_{\alpha} \otimes \varphi_{\alpha}$. We have $\Phi(\alpha x \otimes f+x \otimes f)=u_{\alpha} \otimes \varphi_{\alpha}+u \otimes \varphi$. The fact that $\alpha x \otimes f+x \otimes f$ and the Lemma 2.4 implie that $u_{\alpha} \otimes \varphi_{\alpha}+u \otimes \varphi$ is of rank one. Thus, either $u_{\alpha}$ and $u$ are linearly dependent or $\varphi_{\alpha}$ and $\varphi$ are linearly dependent. Assume that $\varphi_{\alpha}$ and $\varphi$. By absorbing a constant in the first term of the tensor product $u_{\alpha} \otimes \varphi_{\alpha}$ we get $\varphi_{\alpha}=\varphi$. So we have $\Phi(\alpha x \otimes f+z \otimes k)=u_{\alpha} \otimes \varphi+u \otimes \psi . \Phi(x \otimes f+z \otimes k)=u \otimes \varphi+u \otimes \psi$, this and the Lemma 2.4 implie that $x \otimes f+z \otimes k$ is of rank one which holds that $\alpha x \otimes f+z \otimes k$ is of rank one. So $u_{\alpha} \otimes \varphi+u \otimes \psi$ is of rank one. Since $\varphi(u)=1$ and $\psi(u)=0$, the vectors $\varphi$ and $\psi$ are linearly independent. Thus $u_{\alpha}$ and $u$ are linearly dependent and so there exists $\beta \in \mathbf{C}^{*}$ such that $u_{\alpha}=\beta u$. It holds that $\Phi(\alpha x \otimes f)=\beta u \otimes \varphi$. Since $\Phi$ preserves the spectrum we conclude that $\alpha=\beta$.

In the case where $u_{\alpha}$ and $u$ are linearly dependent we get by absorbing a costant in the second term of the tensor product that $u_{\alpha}=u$. We have $\Phi(x \otimes f+w \otimes h)=u \otimes \varphi+v \otimes \varphi$ so by the the Lemma 2.4 the operator $x \otimes f+w \otimes h$ is of rank one. Thus, the operator $\alpha x \otimes f+w \otimes h$ is of rank one. We conclude that $\Phi(\alpha x \otimes f+w \otimes h)=u \otimes \varphi_{\alpha}+v \otimes \varphi$ is an operator of rank one. The fact that $\varphi(u)=1$ and $\varphi(v)=0$ implies that $u$ and $v$ are linearly independent so $\varphi_{\alpha}$ and $\varphi$ are linearly dependent. It holds that there exists $\gamma \in \mathbf{C}^{*}$ such that $\varphi_{\alpha}=\gamma \varphi$. Thus, $\Phi(\alpha x \otimes f)=\gamma u \otimes \varphi$. Since $\Phi$ preserves the spectrum it holds that $\alpha=\gamma$. Finally, we conlude that in this case $\Phi(\alpha x \otimes f)=\alpha \Phi(x \otimes f)$.

Let now paste to the case where $f(x)=0$. We choose $g \in X^{\prime}$ satisfying $g(x)=1$. Then, $\Phi(\alpha x \otimes f)=\Phi(\alpha x \otimes(f+g))-\Phi(\alpha x \otimes g)=\alpha \Phi(x \otimes f)$. 
Now we are ready to prove our main results.

\section{Proof of Theorem 2.1}

The Lemmas 2.1, 2.4 and 2.6 show that $\Phi$ is injective, preserves nilpotent operators of rank one in both directions and is 1-homogeneous on $F_{1}(X)$ respectively. The same hypotheses permit to P. Šemrl in [11] pp:531-532 to conclude that for every $x \in X$ and every $f \in X^{\prime}$ either

$$
\Phi(x \otimes f)=A x \otimes C f,
$$

where $A: X \longrightarrow X$ and $C: X^{\prime} \longrightarrow X^{\prime}$ are linear maps, or

$$
\Phi(x \otimes f)=B f \otimes D x,
$$

where $B: X^{\prime} \longrightarrow X$ and $D: X \longrightarrow X^{\prime}$ are linear maps.

Let us in the situation (2) and consider $z \notin \sigma(T)=\sigma(\Phi(T))$. Define

$$
\begin{aligned}
F_{x, f}(z) & =<(z-T)^{-1} x, f> \\
G_{x, f}(z) & =<(z-\Phi(T))^{-1} A x, C f>.
\end{aligned}
$$

Where $<,>$ denote the duality between $X$ and $X^{\prime}$.

\section{Step 1}

We show that either $G_{x, f} \equiv 0$ or $F_{x, f} \equiv G_{x, f}$ for every $x \in X$ and every $f \in X^{\prime}$.

If $F_{x, f} \neq 1$, then by the Lemma 2.2 , the operator $z-T-x \otimes f$ is invertible in $B(X)$ and so $z-\Phi(T)-A x \otimes C f$ is invertible in $B(X)$, implying that $G_{x, f} \neq 1$. Equivalently, the equation $G_{x, f}=1$ implies $F_{x, f}=1$. But the function $F_{x, f}$ and $G_{x, f}$ are linear in the parameters $x$ and $f$ and so if $G_{x, f}=w \neq 0$, then $F_{x, f}=w$. By analyticity of the two functions, we see that either $G_{x, f} \equiv 0$ or $F_{x, f} \equiv G_{x, f}$.

Let $x_{1} \in X$ and $f_{1} \in X^{\prime}$. If $G_{x_{1}, f_{1}} \not \equiv 0$, then by the foregoing argument $X^{\prime}=\left\{f ; G_{x_{1}, f} \equiv 0\right\} \cup\left\{f ; F_{x_{1}, f} \equiv G_{x_{1}, f}\right\}$ a union of two subspaces. Thus one of the two subspaces is all of $X^{\prime}$. Since $G_{x_{1}, f_{1}} \not \equiv 0$, then $F_{x_{1}, f} \equiv G_{x_{1}, f}$ for every $f \in X^{\prime}$. Similarly we can prove that $F_{x, f} \equiv G_{x, f}$ for every $x \in X$. Finally we have either

$$
<(z-T)^{-1} x, f>=<(z-\Phi(T))^{-1} A x, C f>
$$

or

$$
<(z-\Phi(T))^{-1} A x, C f>=0,
$$


for every $x \in X^{\prime}, f \in X^{\prime}$ and $z \notin \sigma(T)=\sigma(\Phi(T))$.

\section{Step 2}

We prove that only one of the relations (4) or (5) is satisfyed for every $T \in B(X)$.

Assume that there exist $T_{1} \in B(X)$ satisfying (4) and there exist $T_{2} \in$ $B(X)$ satisfying (5). So we have

$$
\begin{gathered}
<\left(z-T_{1}\right)^{-1} x, f>=<\left(z-\Phi\left(T_{1}\right)\right)^{-1} A x, C f> \\
<\left(z-\Phi\left(T_{2}\right)\right)^{-1} A x, C f>=0,
\end{gathered}
$$

for every $x \in X, f \in X^{\prime}$ and $z \notin \sigma\left(T_{1}\right) \cup \sigma\left(T_{2}\right)$. We multiply both relations (6) and (7) by $z$ and let $z$ goes to infinity. We conclude that $<x, f>=<A x, C f>=0$, for every $x \in X$ and erery $f \in X^{\prime}$. This is a contradiction.

\section{Step 3}

We prove that $<(z-T)^{-1} x, f>=<(z-\Phi(T))^{-1} A x, C f>$, for every $T \in B(X)$.

By the second step either (4) is satisfyed for every $T \in B(X)$ or (5) is satisfyed for every $T \in B(X)$. Assume that (5) is satisfyed, then for $T=z-I$ with $z \in \mathbf{C}^{*}$ we have $z \notin \sigma(T)$ and $\langle A x, C f\rangle=0$. Thus, either $A x=0$ or $C f=0$ and by (2) we have $\Phi(x \otimes f)=A x \otimes C f=0$. This conctacictes the invjectivity of $\Phi$.

\section{Step 4}

We prove that $A$ is bounded and $\Phi(T)=A T A^{-1}$ for every $T \in B(X)$.

By the third step we have $\left\langle(z-T)^{-1} x, f>=<(z-\Phi(T))^{-1} A x, C f>\right.$ for every $T \in B(X), x \in X, f \in X^{\prime}$ and $z \notin \sigma(T)=\sigma(\Phi(T))$. By the closed graph theorem, we can easily establish the fact that $A$ and $C$ are bounded. Now we multilply the equality by $z \neq 0$ ( for $z=0$ the problem is trivial ) we hold that $\left.\left\langle z(z-T)^{-1} x, f\right\rangle=<z(z-\Phi(T))^{-1} A x, C f\right\rangle$, thus $<\left(I-\frac{1}{z} T\right)^{-1} x, f>=<\left(I-\frac{1}{z} \Phi(T)\right)^{-1} A x, C f>$. Taking the derivative at $z$ and taking the limit as $z \rightarrow \infty$ we get $\langle T x, f\rangle=\langle\Phi(T) A x, C f\rangle$ for every $x \in X, f \in X^{\prime}$ and $T \in B(X)$. Therefore

$$
T=A^{\prime} \Phi(T) A, \quad \text { where } A^{\prime} \text { is the operator satisfying } C=\left(A^{\prime}\right)^{*} .
$$


We prove that $A$ and $A^{\prime}$ are bijectives. The surjectivity follows easily from (8). Assume that $A^{\prime} y=0$ for some $y \in X$ and take $T_{1} \in B(X)$ such that $\Phi\left(T_{1}\right)=y \otimes g$, where $g \in X^{\prime}$ satisfying $g \neq 0$ and $g(y)=0$. We have $A^{\prime} \Phi\left(T_{1}\right)=0$. By the relation (8) we get $T_{1}=0$ and therefore $y \otimes g=0$, which implies that $y=0$. Thus, $A^{\prime}$ is injective.

The fact that $\Phi(I)=I$ implies that $A^{\prime} A=I$. Since $A^{\prime}$ is bijective we conclude that $A A^{\prime}=I$. So $A^{\prime}=A^{-1}$. Therefore $\Phi(T)=A T A^{-1}$ for every $T \in B(X)$.

\section{Step 5}

Let us go the situation (3) where $\Phi(x \otimes f)=B f \otimes D x$. By a similar proof used in the situation (2), we can show that $B$ and $D$ are bijectives. We prove now that the space $X$ is reflexive. Since $\Phi$ preserves the spectrum we have $(D x)(B f)=f(x)$, this implies that $B$ and $D$ are closed and therefore are bounded. Let $K: X \longrightarrow X^{\prime \prime}$ be the natural embedding of $X$ into $X^{\prime \prime}$. Then the adjoit operator of $D$ (i.e: $D^{*}$ ) is defined at least on the image of $K$ and $D=\left(B^{-1}\right)^{*} K$ where $\left(B^{-1}\right)^{*}$ is the adjoint operator of $B^{-1}$. Thus, $K$ is bijective and bounded. Therefore $X$ is reflexive. We have $<T x, f>=<\Phi(T) B f, D x>$ for every $x \in X, f \in X^{\prime}$ and $T \in B(X)$. So we get $\left\langle x, T^{*} f\right\rangle=<x, B^{\prime} \Phi(T) B f>$, where $B^{\prime}$ is the operator satisfying $D=\left(B^{\prime}\right)^{*}$. Therefore $T^{*}=B^{\prime} \Phi(T) B$ for every $T \in B(X)$. By a similar argument used in the fourth step, we can prove that $B^{\prime}$ and $B$ are bijectives and $B^{\prime}=B^{-1}$. Thus $\Phi(T)=B T^{*} B^{-1}$ for every $T \in B(X)$.

\section{Proof of Corollary 2.1}

By Theorem 2.1 we have either $\Phi(T)=A T A^{-1}$ for every $T \in B(X)$, or $\Phi(T)=B T^{*} B^{-1}$ for every $T \in B(X)$, where $A: X \longrightarrow X$ and $B: X^{\prime} \longrightarrow$ $X$ are linear bounded isomorphisms. It is easy to conclude in the case where $\Phi(T)=A T A^{-1}$ that $\Phi$ is surjective. In the case where $\Phi(T)=B T^{*} B^{-1}$ we have proved in the fifth step of Theorem 2.1 that in this case $X$ is reflexive. Therefore $\Phi$ is surjective.

\section{Remark}

In the case where $X$ is a separable infinite dimensional complex Hilbert space, the proof of our results becomes easier. In fact by a result of Fillmore [6], every operator is a finite sum of square zero operators, and $\Phi$ is surjective. Then we are in the same conditions of B. Aupetit [3]. 


\section{References}

[1] B. Aupetit, Une généralisation du théorème de Gleason-KahaneZelazko pour les algèbres de Banach, Pacific. J. Math 85, pp. 11-17, (1979).

[2] B. Aupetit and H. du Toit Mouton, Trace and determinant in Banach algebras, Studia. Math 121, pp. 115-136, (1996).

[3] B. Aupetit, Sur les transformations qui conservent le spectre, Banach. Algebras 97 (De Gryter, Berlin, pp. 55-78, (1998).

[4] B. Aupetit, A Primer On Spectral Theory (Springer New-York, (1991).

[5] M. Brešar and P. Šemrl, Linear maps preserving the spectral radius, J. Funct. Anal 142, pp. 360-168, (1996).

[6] Fillmore, Sums of operators with square-zero, Acta. Sci. Math. Szeged. 28, pp. 285-288, (1967).

[7] A. A. Jafarian and A.R. Sourour, Spectrum preserving linear maps, J. Funct. Anal 66, pp. 255-261, (1986).

[8] M. Omladič and P. Šemrl, Spectrum preserving additive maps, Linear. Algebras. Appl 153, pp. 67-72, (1991).

[9] W. Rudin, Functional Analysis.

[10] P. Šemrl, Spectrally bounded linear maps on $B(H)$, Quat. J. Math. Oxford (2) 49, pp. 87-92, (1998).

[11] P. Šemrl, Linear maps that preserve the nilpotent operators, Acta. Sci. Math (szeged) 61, pp. 523-534, (1995).

[12] S. Sakai, $C^{*}$-Algebras and $W^{*}$-Algebras (Springer,New-York, (1971).

[13] A.R. Sourour, Invertibility preserving linear maps on $\mathcal{L}(X)$, Trans. Amer. Soc 348, pp. 13-30, (1996). 


\section{Mustapha Ech-chérif El Kettani}

Département de Math et Informatique

Faculté des sciences Dhar El Mehraz

B.P 1796 Atlas Fès Maroc

e-mail : melkettani@caramail.com

\section{El Houcine El Bouchibti}

Département de Math et Informatique Faculté des sciences Dhar El Mehraz

B.P 1796 Atlas Fès Maroc

e-mail : elbouchibti@hotmail.com 